\title{
Ageing and the Spatiotemporal Discrimination Performance of the Visual System
}

\author{
DONALD W. KLINE \\ Calgary, Alberta
}

\begin{abstract}
Summary
Visual ageing is generally associated with a decline in spatial vision for stationary stimuli, especially those which are of high spatial frequency. Some, but probably not all of this loss can be attributed to apparently normal age-related changes in the ocular media that attenuate and scatter incident light. The loss in the temporal resolving capacity of the senescent visual system is even more prominent and appears to be due almost completely to neural mechanisms, including alterations in the functioning of both the 'transient' and 'sustained' types of visual channel. The losses with age in both spatial and temporal vision are highly consistent with the type and degree of visual problems elderly persons report that they experience in carrying out daily tasks. Further research will be needed to determine the mechanisms which underlie these changes as well as to determine the degree to which they can be remedied.
\end{abstract}

\section{Spatial Resolution, Ageing and Retinal Illuminance}

Normal age-related changes in the optic media of the eye attenuate, scatter, and alter the spectral composition of incident light contributing to age-related losses in acuity, sensitivity and task performance that are particularly evident under conditions of poor illumination. Although some of the attenuation and scatter can be attributed to alternations in the cornea and vitreous humour, the most functionally important of the age-related optic media changes appear to be the increased opacity of the lens and the reduced diameter of the pupil. ${ }^{1.2}$

Static Visal Acuity (SVA). There is, on average, a significant decrease in static visual acuity. ${ }^{3}$ The primary pathological processes involved in the age-related decline in acuity are in decreasing order of importance: cataract, age-related maculopathy, retinal pathology including diabetic retinopathy and open angle glaucoma. ${ }^{4}$ The normal age-related reduction in retinal illumination also appears to play a role in the loss of acuity. Weston ${ }^{5}$ found that increases in target illumination produced greater improvements in the accuracy of Landolt gap identification in old persons than young ones, although acuity of the old observers never reached the level of the young. Similarly, increased contrast can improve markedly the target letteridentificationperformance among older persons. ${ }^{6}$

Findings such as these have significant implications for the predictive validity of SVA measures as they have been traditionally

From: Department of Psychology, University of Notre Dame, Notre Dame, and Visiting Scholar, Cresap Neuroscience Lab, Northwestern University.

Supported by a Research Career Development Award from the National Institute on Aging (No. K04 AG00199) and a Travel Grant from the Institute for Scholarship in the Liberal Arts, University of Notre Dame.

Correspondence to: Donald W. Kline, Department of Psychology, University of Calgary, Calgary, Alberta, Canada T2N 1N4. 
assessed especially among older persons. In SVA, a high-contrast optotype is presented in 'good' illumination (5 to $20 \mathrm{FtC}$ is often recommended). Such conditions are useful for refraction and usually appropriate for predicting the acuity of younger persons under bright daylight conditions since young adults show little improvement in acuity with increases in illuminance past 10 to $20 \mathrm{FtC}$. This is not the case, however, for older persons whose retinal illuminance is considerably below that of younger observers. ${ }^{7}$ As a result, the SVA of older persons under daylight conditions may often be seriously underestimated by tests administered under 'recommended' lighting levels. The older the observer, the more serious this problem is likely to be. The predictive validity of such tests for most older persons might well be improved simply by increasing the light at which the tests are given, perhaps by a factor of at least 10 or 20 . Relatedly, since the acuity of older persons falls disproportionately in dim illumination, high-illumination SVA provides an inadequate basis for determining the acuity of older persons on tasks carried out under low illumination. Shinar, ${ }^{8}$ for example, found that acuity under low illumination was a singificant predictor of overall auto accident rate and further, was superior to high illumination acuity in this regard. This underscores the importance of testing acuity task conditions representative of those in which the acuity will be needed, especially where elderly persons are concerned. While elevations of contrast and/or illumination can enhance the acuity of older persons, usually it will not make them equal to their 20-year-old colleagues. ${ }^{9}$ It should also be noted, however, that there are great individual differences in this and other tested visual functions; it is not uncommon to see older persons in the laboratory with SVAs of $20 / 20$ or even $20 / 16$.

Contrast Sensitivity. The contrast sensitivity function (CSF) provides a comprehensive statement of spatial vision abilities by determining the minimum contrast needed to detect stimuli (usually bar gratings) which vary in spatial frequency expressed in cycles per degree of visual angle. Age studies of the static CSF show a marked decline in contrast sensitivity at intermediate and high spatial frequencies (i.e. above 3 to $4 \mathrm{c} / \mathrm{deg}) \cdot{ }^{10.11}$ Further, if retinal illuminance was roughly equalised ${ }^{11}$ for 20-year-olds and 60-year-olds, the sensitivity difference was reduced greatly, although not eliminated. This indicates that some of the loss was due to the decrease with age in retinal illumination, and by implication, part of it may be attributable to photoreceptor/neural changes. Owsley, Gardner, Sekuler and Lieberman ${ }^{12}$ found that contrast sensitivity loss for intermediate and high spatial frequencies was comparable for old subjects with 'normal' lenses and those with intraocular lens (IOL) implants, demonstrating that normal age-related increases in lenticular opacity play little role in the age loss of contrast sensitivity. Pupillary dilatation with drops, however, did reduce the high spatial frequency loss by about 40 per cent, indicating that in addition to neural change, the decrease in retinal illuminance associated with senile miosis was a contributing factor.

Age, Dark Adaptation and Glare. Studies of dark adaptation and age find a marked elevation in final adapted threshold, an elevation which is particularly marked past the age of 60 years. Testing subjects aged 20 to 60 years, McFarland and Fisher ${ }^{13}$ found a correlation of 0.89 between age and final adaptation level; the intensity of the test light at threshold had to be approximately doubled for each 13 years of age. In fact, the relationship between age and final adapted threshold was so strong that it could be used to predict a subject's age within three years. Fairly similar results have been found in other studies employing subjects ranging even more broadly in age. They indicate that from the 20 son there is approximately an increase in threshold of $1.7 \mathrm{log}$ units to the $70 \mathrm{~s}$ and $2.5 \log$ units to the $80 \mathrm{~s}$. There is good reason to believe that a large portion of this elevation can be attributed to senile miosis and the increased opacity of the lens, and that a smaller part may be due to metabolic changes in the retina. ${ }^{3}$ The actual rate at which dark adaptation occurs does not appear to change with age. The apparent reduction in adaptation rate seems to reflect only the delay in reaching a particular adaptation level that reflects the upward displacement of the older observer's entire adaptation function. ${ }^{14}$

Glare, which results when light that is overly bright or inappropriately directed reduces 
visual effectiveness, appears to be a greater problem for old observers than young ones. Wolf ${ }^{15}$ found that the inability to identify a target under veiling glare increased with age, except in older eyes from which the lens had been removed, suggesting that the age-related susceptibility to veiling glare is attributable largely to changes in the lens. The contrast reduction effects of veiling glare are likely to be most disadvantageous to older pesons under conditions of low illumination.

Performance on Everyday Tasks. There is a small but increasing research literature that indicates that the decline with age in retinal illuminance can limit performance on everyday tasks, and that appropriate manipulation of lighting conditions can often compensate to some degree for this limitation. Hughes and McNelis ${ }^{16}$ compared the performance of two age groups (19-27 and 46-57) on a visual search task under three levels of illumination (536, 1076 and 1614 lux). Performance times decreased as a function of increased light for both groups, but to a greater degree for the older group. In addition, the older group reported greater visual comfort at the two higher levels. A similar result was found in a study ${ }^{17}$ which examined the lighting requirements for properly-refracted older workers (age 17 to 65 years). The observer's task was to read 10 seven-letter words under different conditions of illumination (from 20 to $80 \mathrm{Ft}$.C). If provided with a sufficient illumination advantage, the visibility of the stimuli to the older subjects equalled that of the youngest group.

Although the data from such research provide badly needed estimates of the effects of ageing of the ocular media on laboratory-type tasks, they do not assess the consequences of visual changes on the daily lives of older persons. Kosnik, Rasinski, Kline, Winslow and Sekuler ${ }^{18}$ used a questionnaire to ask several hundred adults of different ages (from the 20s to the 90s), and without serious eye disease, to report the frequency of impact of visual problems on performance of their daily activities using a 4-point rating scale. Among other findings, problems associated with seeing under conditions of poor illumination were considerably more common among the elderly. Specifically, they reported significantly greater difficulty on such tasks as adjusting to dim illumination, seeing in dimly lit rooms, seeing at dusk, distinguishing between dark colours (such as black and navy blue) and in night driving, that their instrument panels were too dimly illuminated.

The implications of age-related ocular media change are in many cases quite clear. Poorly-lit environments will differentially handicap the performance of older persons, and their problems are likely to be exacerbated by visual environments or tasks that are complex or cluttered, and/or require attending to small size print or detail. Unfortunately, traditional visual measures, such as static visual acuity, do not appear adequate for predicting in whom, under what conditions and to what degree many of these problems will occur. The exent to which these problems vary for different people of different ages under different task conditions, and how they can best be identified in advance by more appropriate visual testing will need to be determined by future research.

\section{Age and Temporal Processing}

\section{'All is flux, nothing stays still.' (Heraclitus)}

From the time of Heraclitus' observation, the world has changed so as to tax increasingly the visual system's ability to extract order and meaning from the 'flux'. Perhaps the most prominent functional decline in the older visual system is in its ability to detect temporal change, or to resolve detail in that change. Temporally contiguous visual stimuli that would be seen as separate by young observers are often seen as fused or 'smeared' by older persons. This phenomenon is observable in such diverse age-related phenomena as the lowered CFF threshhold, the extended susceptibility to the effects of backward visual masking, the durability of visual after-images, the protracted period over which form can be integrated, the diminished ability to discriminate motion and in the decline in dynamic visual acuity (DVA). ${ }^{1}$ Although no single mechanism appears to account for the diminished temporal processing capacity of the senescent visual system, it is clear that changes in the ocular media explain little of this change, that most of it is 'neural' in its origins. Among these significant contributors there appear to be 
changes in the temporal response characteristics of both types of visual channels, the transient and sustained, and in the effectiveness of the oculomotor system responsible for smooth pursuit eye movements.

Dynamic Visual Acuity (DVA). Many daily tasks, like walking, driving or operating machinery involve relative motion between observer and target. A visual test involving motion, such as dynamic visual actuity (DVA) would appear more suitable than SVA for assessing visual fitness for tasks of this type, especially when the participant is elderly. In fact, DVA, the ability to discriminate detail (e.g. Landolt Cs, checkerboards, etc) during the ocular pursuit of a moving target, is a much better predictor of automotive driving record than is SVA, especially for older drivers. ${ }^{23,24}$ There are a variety of reasons for this. Although SVA sets the upper limit for DVA, the relationship between the two measures falls progressively as target velocity is increased. ${ }^{25.26}$ This appears to be due primarily to increased movement of the target image over the retina as the velocity gain in smooth pursuit eye movements fails to match that occurring in the target. ${ }^{27}$ That is, DVA appears to depend on the accuracy of control of eye speed, not on lack of eye velocity per se. Given that smooth pursuit eye movements are diminished in their effectiveness with age ${ }^{28.29}$ the progressive decline in DVA with age that is particularly prominent at high target velocities $^{30,31,32}$ is not unexpected.

Since several factors that affect DVA $^{33}$ are also strongly age related, DVA might be particularly sensitive to individual age-related changes in visual fitness. Firstly, there is a significant decline with age in retinal illumination $^{7}$ and DVA responds to illumination change over a much broader range than does SVA. Miller ${ }^{34}$ found that while SVA threshholds were essentially asymptotic at $10 \mathrm{ft}-\mathrm{c}$, DVA threshholds at $120 \% \mathrm{sec}$ were still improving with increased illumination at $125 \mathrm{ft}-\mathrm{c}$. Also, the beneficial effect of increased illumination was greater at higher target velocities, which is where the greatest age differences are observed. Secondly, DVA improves with increased target contrast, ${ }^{35}$ and it is known that older observers need higher contrast levels to detect intermediate and high- spatial-frequency targets. ${ }^{10,11}$ Thirdly, DVA improves with longer target durations up to $11.0 \mathrm{sec}^{36.37}$ and thus, it should be more sensitive to the general slowing that appears to be a universal characteristic of ageing ${ }^{38}$ than are static tests. In short, the responsivity of DVA to manipulations of illumination, contrast and duration, suggest it would be highly useful as a screening tool, particularly with older persons. Systematic research, however, will be needed to determine the degree to which age differences in DVA threshholds are in fact a function of the stimulus parameters which affect DVA. It will also be important to determine the extent to which the DVA threshholds are as amenable to change through practice ${ }^{39}$ and/or free versus fixed-head presentation ${ }^{37}$ in older observers as they are in younger ones.

Motion Discrimination and the Visual Channels. What mechanism(s) can account for the relatively poor temporal processing of the older visual system? Kline and Schieber ${ }^{20}$ hypothesised and there is some evidence ${ }^{10,21,22}$ to support a 'transient/sustained shift' notion, that at least some of the age-related loss of temporal resolution can be attributed to the diminished effectiveness of the transient visual channels. Certainly, a visual system having undergone transient channel decline, with an associated decrease in inhibition of sustained channels would mirror many of the changes seen among older persons carrying out visual tasks: slowed response speed, poor detection of stimulus change and the protracted persistence of the neural effects of visual stimulation. Recent research does in fact suggest an age-related impairment of the transient channels. However, it also points to changes in the temporal resolving power of higher-spatialfrequency sensitive sustained channels.

Owsley, Sekuler and Siemsen ${ }^{11}$ found that the motion-induced enhancement of sensitivity to a low spatial frequency grating (1 $\mathrm{c} / \mathrm{deg}$ ) that is typically observed in young observers was very much diminished in adults over 60 years of age. Further, the age difference in enhancement was substantially greater when the grating was drifted quickly $(4.3 \mathrm{deg} /$ sec) than when it drifted slowly $(1.1 \mathrm{deg} / \mathrm{sec})$. This impairment of temporal processing could not be accounted for by the reduction in retinal illumination in the aged eye. 


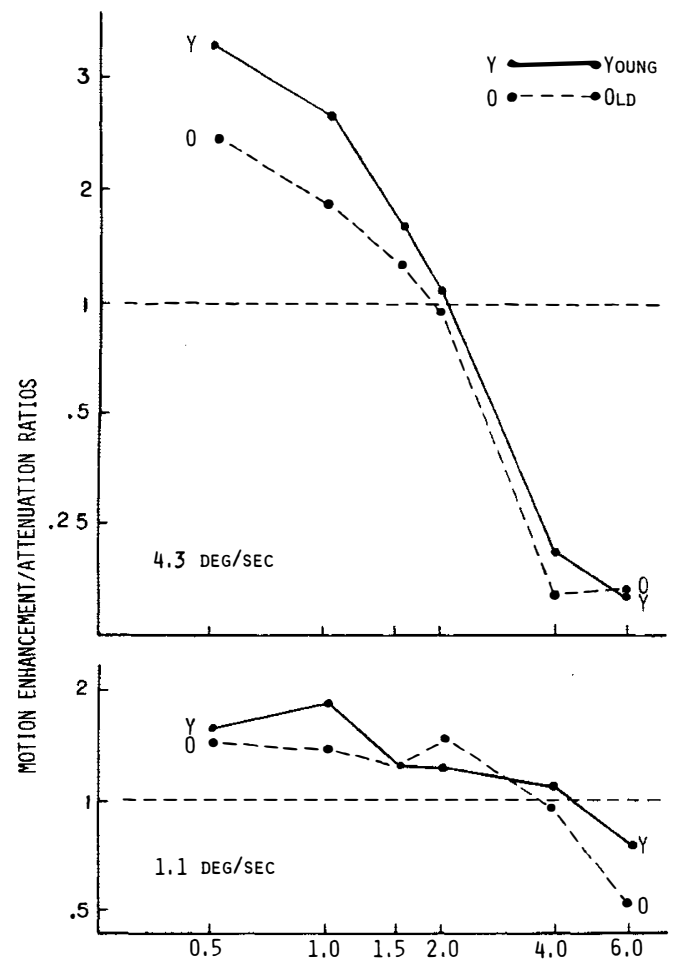

SPATIAL FREQUENCY (C/DEG)

Fig. 1. Motion enhancement/attenuation ratios (static/movement contrast threshold) as a function of age, spatial frequency and movement rate (Kline, Schieber, Abusamra, and McCoy, 1986).

Kline, Schieber, Abusamra and McCoy, ${ }^{21}$ examined age differences in the spatial bandwidth of the effect of motion in enhancing contrast sensitivity. The contrast sensitivity functions for young and old adults with excellent acuity were compared for static and dynamic $(0,1.1$ and $4.3 \mathrm{deg} / \mathrm{sec})$ sine-wave gratings ranging from 0.5 to $6.0 \mathrm{c} / \mathrm{deg}$. Motion enhancement or attenuation ratios were calculated for each observer. Following Owsley, Sekuler and Siemsen, ${ }^{11}$ the contrast threshhold at each spatial frequency for either the slow or fast movement condition was divided by the corresponding threshhold in the static condition. Ratio values greater than 1.0 indicate that motion enhancement sensitivity; ratios less than 1.0 indicate that motion attenuated it. As can be seen in Figure 1, motion enhancement of sensitivity was considerably more robust among younger subjects in the low spa- tial frequency range. By this measure, motion enhancement was significantly greater at 1 $\mathrm{c} / \mathrm{deg}$ and approached significance at $0.5 \mathrm{c} / \mathrm{deg}$, a finding consistent with age-related transient channel decline. In addition, however, sensitivity attentuation was greater for the old observers at both $6 \mathrm{c} / \mathrm{deg}$ in the slow movement condition and at $4 \mathrm{c} / \mathrm{deg}$ in the rapid movement condition. One mechanism that might explain this latter result is an age-related loss in the effectiveness of integration within the high spatial frequency sustained channels. An increase with age in the critical duration from 155 to 232 msec was observed when estimated from the point of peak contrast sensitivity for fast-moving sinusoidal gratings. ${ }^{21}$ Further, there is evidence of disproportionate slowing in response to the higher spatial frequencies mediated by these channels as indicated by greater age differences in response speed to high spatial frequencies ${ }^{10}$ (Fig. 2).

In a second study, we tried to determine the mechanism(s) underlying the age difference in motion enhancement at low spatial frequencies by examining age differences in the attenuation of contrast sensitivity $(4.3 \mathrm{deg} / \mathrm{sec})$ low spatial frequency gratings ( 0.5 to $2 \mathrm{c} / \mathrm{deg})$ following extended adaptation to a prior stationary or drifting $1.0 \mathrm{c} / \mathrm{deg}$ grating. ${ }^{40} \mathrm{~A}$ striking pattern of age differences in the spatial specificity of the adaptation effect emerged. Adaptation was narrowly turned around the

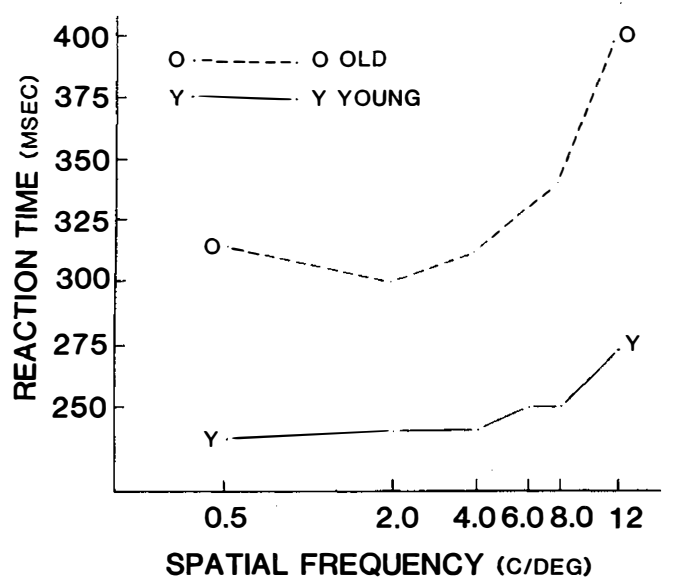

Fig. 2. Age differences in reaction time as a function of spatial frequency (Kline, Schieber, Abusamra, and Coyne, 1983). 


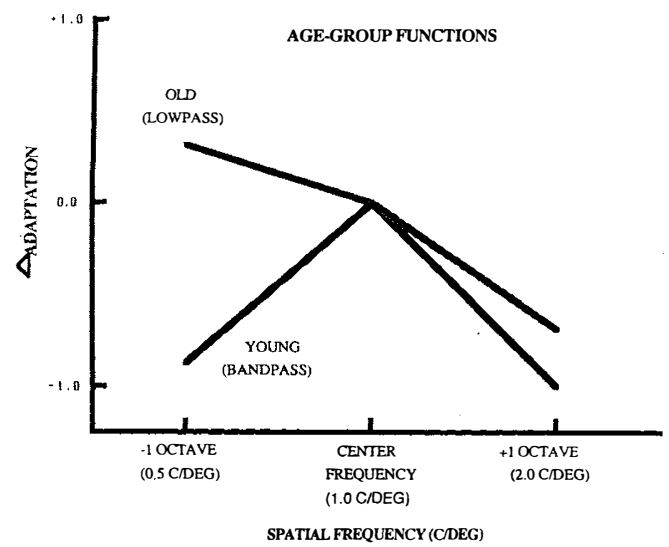

Fig. 3. Relative magnitude of adaptation at one octave above and below the frequency of the adaptation grating when the young and old groups where

$\Delta$ adaptation $=$ rel. threshold elevation ${ }_{1.0} \mathrm{c}_{\mathrm{deg}}$-rel. threshold elevation $_{+1-1}$ octave

rel. threshold elevation ${ }_{1.0}$ CIDEG

centre frequency of the adapting stimulus in the young subjects, but broadly turned in old subjects. That is, the adaptation effects in the young and old subjects could be well represented as 'bandpass' and 'lowpass' respectively (Fig. 3).

Further, these appeared to be true individual effects, not merely the reflection of group averages; 8 of 8 young subjects could be characterised as 'bandpass' and 7 of 8 old as 'lowpass'. It is as though the low spatial frequency channels lose their tuning specificity and become 'linked' so as to function more globally. Or alternatively, they are lost revealing a more 'primitive' process. Whatever the specific underlying mechanisms for age differences in dynamic CSFs, the lesson for visual testing is clear: age differences in visual sensitivity vary greatly depending on both the rate of motion and the spatial characteristics of the display.

In terms of possible remediation of age declines in visual processing, Ball and Sekuler ${ }^{41}$ found that while young observers were superior in discriminating one direction of motion from another, practice steadily improved the performance of both groups equally. Further, the gains made by both age groups were retained for at least one month following practice. It remains to be seen if practice or other remedial interventions can improve other aspects of temporal processing.

These general findings are in strong agreement with what old people tell us about changes in their vision, and which appear to have significant effects on their daily lives. When Kosnik and his colleagues ${ }^{18}$ surveyed several hundred people of different ages, the most salient of several important problems that emerged in their responses was the difficulty that they had with items relating to slowed visual processing speed. These problems included taking more time to do visual tasks in general, reading more slowly, difficulty reading scrolling credits on television, and problems reading moving signs such as those on passing trucks (a deficit quite similar to that occurring in DVA).

In conclusion, there are declines with age in the resolution of spatial detail that become particularly prominent when the visual display is modulated temporally. Although, alterations in the operating characteristic of the visual channels appear to be involved in this change, future research will be needed to determine the specific underlying neural mechanisms. The recognition that current laboratory findings in this regard are highly consistent with the visual changes that elderly persons report as significant problems in their daily lives should give impetus to this effort.

\section{References}

${ }^{1}$ Kline DW, Schieber F: Vision and aging. In: Birren JE and Schaie KW, eds. Handbook of the psychology of aging 2nd ed. New York: Van Nostrand Reinhold 1985; 296-31.

${ }^{2}$ Sekuler R, Kline D, Dismukes K, eds: Aging and Human Visual Function. New York: AR Liss 1982; 350 .

${ }^{3}$ Pitts DG: The effects of aging on selected visual functions: dark adaptation, visual acuity, stereopsis, and brightness contrast. In: Sekuler R, Kline D, Dismukes K, eds. Aging and Human Visual Function. New York: AR Liss 1982; 131-59.

${ }^{4}$ Greenberg DA, Branch LG: A review of methodologic issues concerning incidence and prevalence of visual deterioration in elders. In: Sekuler R, Kline D, Dismukes K, eds. Aging and Human Visual Function. New York: AR Liss 1982; 279-96.

${ }^{5}$ Weston HC: On age and illumination in relation to visual performance. Trans. Illum Eng. Soc. 1949; 14: 281-97.

${ }^{6}$ Blackwell OM, Blackwell HR: Visual performance 
data for 156 observers of various ages. J. Illum. Eng. Soc. 1971; 1: 3-13.

${ }^{7}$ Weale RA: Retinal illumination and age. Trans. Illum. Eng. Soc. 1961; 26: 95-100.

${ }^{8}$ Shinar D: Driver visual illumination: Diagnosis and treatment. Indiana University, Institute for Research in Public Safety, Department of Transportation contract DOT-HS-5-1275 1977; 203.

${ }^{9}$ Richards OW: Effects of luminance and contrast on visual acuity, ages 16 to 90 years. Am. J. Optom Physiol Opt. 1977; 54: 178-84.

${ }^{10}$ Kline DW, Schieber F, Abusamra LC, Coyne AC: Age, the eye, and visual channels: Contrast sensitivity and response speed. J. Gerontol. 1983; 38: 211-6.

${ }^{11}$ OwsleyC, Sekuler R, Siemsen D:Contrastsensitivity throughout adulthood. Vision Res. 1983; 23: 68999.

${ }^{12}$ Owsley C, Gardner T, Sekuler R, Lieberman H: Role of crystalline lens in the spatial vision loss of the elderly. Invest. Ophthalmol. Vis. Sci. 1985; 26: 1165-70.

${ }^{13}$ McFarland RA, Fisher MB: Alterations in dark adaptation as a function of age. J. Gerontol. 1955; 10: 424-8.

14 Weale RA: On the eye. In: Welford AT, Birren JE, eds. Behavior, Aging and the Nervous Systems. Springfield: CC Thomas 1965; 307-25.

15 Wolf E: Glare and age. Arch. Ophthalmol. 1960; 60: 502-14.

${ }^{16}$ Hughes PC, McNelis JF: Lighting productivity and the work environment. Paper presented at the Annual Illuminating Engineering Society Technical Meeting, Denver, CO, August 1978.

${ }^{17}$ Guth SK, Eastman AA, McNelis JF: Lighting requirements for older workers. Illum. Eng. 156; 51: 656-60.

${ }^{18}$ Kosnik W, Rasinski K, Kline D, Winslow L, Sekuler $\mathrm{R}$ : Age-related visual changes in everyday life (1986 under review).

${ }^{19}$ Kline DW, Schieber F: Visual persistence and temporal resolution. In: Sekuler R, Kline D, Dismukes K, eds. Aging and Human Visual Function. New York: AR Liss 1982; 231-44.

${ }^{20}$ Kline DW, Schieber F: Visual aging: a transient/sustained shift? Percept. Psychophys. 1981; 29: 181-2.

${ }^{21}$ Kline DW, Schieber F, Abusamra LC, McCoy L: Contrast thresholds for moving gratings in older adults: Evidence for a transient deficit (1986 under review).

22 Sturr J, Church KL, Taub HA: Early light adaptation. in young, middle-aged and older observers. Percept Psychophys. 1985; 13: 455-8.

${ }^{23}$ Burg A: The relationship between vision test scores and driving record: General findings (report 67-24). Los Angeles: University of California, Department of Engineering 1967.

${ }^{24}$ Hills BL, Burg A: A reanalysis of California driver vision data: General findings (report LR768).
Department of the Environment/Department of Transport, TRRL, Crowthorne, Berks 1977.

${ }^{25}$ Burg A, Hulbert SF: Dynamic visual acuity as related to age, sex and static acuity. J. Appl. Psychol. 1961; 45: 111-16.

${ }^{26}$ Miller JW, Ludvigh EJ: Dynamic visual acuity when the pursuit is in the vertical plane. NSAM-563, Pensacola, FL: Naval School of Aviation Medicine 1953.

${ }^{27}$ Brown B: Dynamic visual acuity, eye movements and peripheral acuity for moving targets. Vision Res. 1972; 12: 305-21.

${ }^{28}$ Sharpe J, Sylvester T: Effects of aging on horizontal smooth pursuit. Invest. Ophthalmol. and Vis. Sci. 1978; 17: 465-8.

${ }^{29}$ Spooner J, Sakala S, Baloh R: Effect of aging on eye tracking. Arch. Neurol. 1980; 37; 575-6.

${ }^{30}$ Burg A: Visual acuity as measured by dynamic and static tests: A comparative evaluation. J Appl Psychol. 1966; 50: 460-6.

${ }^{31}$ Farrimond T: Visual and auditory performance variations with age: Some implications. Austral. J. Gerontol. 1967; 19: 193-201.

32 Reading VM: Visual resolution as measured by dynamic and static tests. Pflug. Arch. 1972; 333: 17-26.

${ }^{33}$ Morrison TR: A review of dynamic visual acuity. Naval Medical Research and Development Command, Naval Aerospace Medical Research Laboratory: Pensacola, Florida 1980, _.3.

${ }^{34}$ Miller JW: The effect of altered illumination on visual acuity measured during ocular pursuit. NSAM573. Pensacola, FL: Naval School of Aviation Medicine 1956.

${ }^{35}$ Mayyasi AM, Beals RP, Templeton AE, Hale PN Jr: The effects of ambient illumination and contrast on dynamic visual acuity. Am. J. Optom. Arch. Amer. Acad. Optom. 1971; 48: 844-8.

${ }^{36}$ Brown B: the effect of target contrast variation on dynamic visual acuity and eye movements. Vision Res. 1972; 12: 1213-24.

${ }^{37}$ Crawford WA: The perception of moving objects. I. Ability and visual acuity. Memo 150a. Farnborough, England: RAF Flying Personnel Research Committee 1960.

38 Welford AT: Motor performance. In: Birren JE, Schaie KW, eds. Handbook of the psychology of aging. New York: Van Nostrand Reinhold 1977; 450-96.

${ }^{39}$ Miller JW, Ludvigh EJ: An analysis of certain factors involved in the learning process of dynamic visual acuity for 1000 naval aviation cadets. NSAM-574. Pensacola, FL: Naval School of Aviation Medicine 1957.

${ }^{40}$ Schieber F, Kline DW, Lyman BJ: Adult age differences in the bandwidth of adaptation for moving, low spatial frequency sine-waving gratings. Paper presented at the Association for Research in Vision and Ophthalmology Annual Meeting, Sarasota, Florida 1986.

${ }^{41}$ Ball K, Sekuler R: Improving visual perception in older persons. J. Gerontol. 1986; 41: 176-82. 